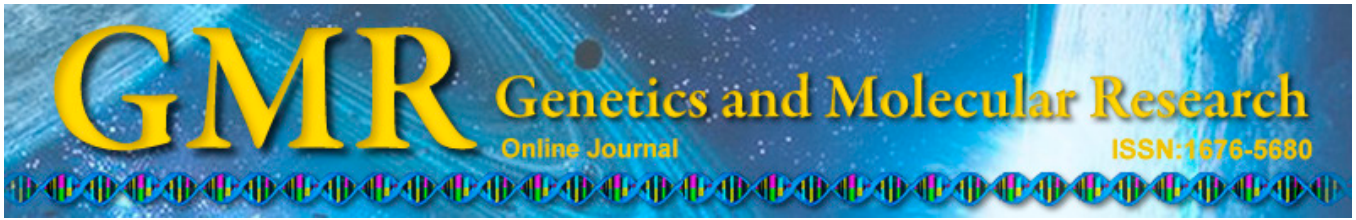

\title{
Expression of aquaporins 1 and 3 in degenerative tissue of the lumbar intervertebral disc
}

\author{
S.B. Li ${ }^{1}$, K.S. Yang ${ }^{1}$ and Y.T. Zhang ${ }^{2}$ \\ ${ }^{1}$ Department of Orthopedics, Affiliated Hospital of Dali University, Dali, \\ Yunnan Province, China \\ ${ }^{2}$ Department of Orthopedics, Zhoushang TCM and Orthopedics Union Hospital, \\ Zhoushang, Zhejiang Province, China \\ Corresponding author: K.S. Yang \\ E-mail: yangks126@126.com
}

Genet. Mol. Res. 13 (4): 8225-8233 (2014)

Received June 14, 2014

Accepted August 18, 2014

Published October 8, 2014

DOI http://dx.doi.org/10.4238/2014.October.8.4

ABSTRACT. Lumbar intervertebral disc degeneration is induced by
multiple factors, but few studies have examined the effects of aquaporins
on this process. We compared the expression levels of aquaporins 1 and
3 in normal and degenerative lumbar intervertebral discs. Fifteen normal
and 15 degenerative lumbar intervertebral disc tissues were excised from
lumbar burst fracture patients during orthopedic operations at the Dali
College subsidiary hospital. Tissues were stained with hematoxylin-
eosin and the expression levels of aquaporins 1 and 3 were measured by
immunohistochemistry. Hematoxylin-eosin-staining results illustrated
that the structures of the intervertebral disc tissues from the control
group were clear, with distinct collagen fiber shapes and slight edema
but without mucoid degeneration. Structures of the intervertebral
disc tissues from the disease group were obscure and disordered with
hyperplastic collagen fibers and tissues of severe inflammatory edemas
with necrosis mucoid degeneration. Immunohistochemistry results
demonstrated that the average absorbances of aquaporins 1 and 3 in the
disease group were significantly lower than those in the control group
(P $<0.01$ ), suggesting that the reduction of aquaporins 1 and 3 may be a 
factor resulting in lumbar intervertebral disc degeneration.

Key words: Degenerative tissue; Expression of aquaporin; Lumbar intervertebral disc

\section{INTRODUCTION}

Lumbago and scelalgia induced by lumbar intervertebral disc degeneration are common, and the economic and human resource losses caused by lumbar intervertebral disc degeneration are substantial. Lumbar intervertebral disc degeneration has been extensively investigated worldwide. The mechanism involves biological, genetic, biomechanical, and nutritional factors (Sobajima et al., 2004; Chan et al., 2006; Li et al., 2008; Yuan et al., 2011). A decrease in water content is one characteristic of lumbar intervertebral disc degeneration. The water content of intervertebral disc nucleus pulposus during childhood is greater than $80 \%$, whereas this value is $70 \%$ at older ages. Therefore, water content is an important factor underlying the mechanisms of lumbar intervertebral disc degeneration.

Water is important for maintaining cell physiological functions. Water enters cells through 3 routes: diffusion, permeation, and transportation channels. As water is transported, nutrition and metabolism products are also transported. Water transmembrane transportation is important for maintaining normal metabolism in cells. Molecular biology studies revealed that aquaporins change during the course of various diseases (Yang et al., 2001; Takata et al., 2004; Nesic et al., 2006). Aquaporin 1 is a transport channel for water and is widely expressed in systemic cells. Aquaporin 3 can also transport glycerol. Aquaporins 1 and 3 may participate in lumbar intervertebral disc degeneration (Richardson et al., 2008; Cao et al., 2011; Wang and Zhu, 2011). The effects of aquaporins 1 and 3 on lumbar intervertebral disc degeneration were examined by detecting their expression change patterns in lumbar intervertebral disc tissue.

\section{MATERIAL AND METHODS}

\section{Materials}

Surgery specimens were provided by the Orthopedic Department of the Dali College subsidiary hospital.

\section{Control group}

Fifteen cases of lumbar intervertebral disc tissues with main $\mathrm{L}_{1-2}$ and $\mathrm{L}_{2-3}$ lumbar intervertebral discs were obtained from lumbar burst fracture patients (11 males, 4 females; mean age of 28.5 years). The lumbar intervertebral disc degeneration degree was determined using the Thompson classification method (Thompson et al., 1990), and discs were defined as degenerated when below degree II and were validated as normal by tissue pathology examination.

\section{Disease group}

Fifteen cases of degraded lumbar intervertebral disc tissues with main $\mathrm{L}_{4-5}, \mathrm{~L}_{5}-\mathrm{S}_{1}$ lumbar 
intervertebral discs were removed from patients with lumbar disc herniation and lumbar spinal stenosis ( 9 males, 6 females; mean age of 47.5 years). Lumbar intervertebral disc degeneration degree was determined using the Thompson classification method (Thompson et al., 1990), and discs were defined as degenerated above degree III and validated as degenerative lumbar intervertebral discs by tissue pathology examination.

\section{Reagents}

Rabbit anti-human aquaporin 1 and 3 monoclonal antibodies were purchased from the Beijing ZSGB Biotechnology Company (Beijing, China).

\section{Specimen treatment}

Lumbar intervertebral disc tissues with volumes of $1.0 \times 1.0 \times 0.5 \mathrm{~cm}$ were selected and fixed with $10 \%$ formalin solution for $24 \mathrm{~h}$. After washing with water, tissues were dehydrated from low to high concentrations using $30,70,85$, and $95 \%$ (volume rate) alcohol and ethanol successively for $1 \mathrm{~h}$ per condition. Tissues were soaked in a mixture solution containing 50/50 ethanol and butanol for $1 \mathrm{~h}$ and transferred to butanol for soaking for $1 \mathrm{~h}$. The transparent agent was replaced with paraffin wax. Tissue materials after wax soaking were placed in paraffin liquid to form a paraffin block containing the tissue block with a serial number. Blocks were sliced at a thickness of $5 \mu \mathrm{m}$.

\section{Hematoxylin-eosin (HE)-staining procedure}

The HE-staining procedure was performed according to the protocol described previously (Dimcevici et al., 2014).

\section{Immunohistochemistry-staining procedure (SP method)}

Paraffin sections that were deparaffinized in water were washed with distilled water and soaked in phosphate-buffered saline (PBS) for $5 \mathrm{~min}$. Pancreatin was added dropwise (diluted 1:3) and the sample was incubated at $37^{\circ} \mathrm{C}$ for $10 \mathrm{~min}$ for antigen retrieval to eliminate endogenous peroxidase activity and washed with PBS 3 times for 2 min each time. The sample was sealed with 5\% (volume rate) normal goat serum and incubated at room temperature for $15 \mathrm{~min}$ with serum, which was discarded and without washing. Next, $50 \mu \mathrm{L}$ rabbit anti-human aquaporin $(1: 400)$ and aquaporin 3 (1:400) monoclonal antibodies were added separately and incubated at $4{ }^{\circ} \mathrm{C}$ overnight. PBS was added to replace the primary antibody in the negative control. After incubating overnight, the sample was placed at room temperature for $30 \mathrm{~min}$ and washed with PBS 3 times for 2 min each time. Next, 50 $\mu \mathrm{L}$ biotin-labeled anti-rabbit $\operatorname{IgG}(1: 150)$ was incubated with the sample at $37^{\circ} \mathrm{C}$ for $15 \mathrm{~min}$ and washed with PBS 3 times for 2 min each time. Staining by DAB was observed under a microscope to determine the control coloration degree. The sample was washed with water for $15 \mathrm{~min}$, restained with hematoxylin for $2 \mathrm{~min}$, subjected to hydrochloric acid alcohol differentiation, washed with water for 10-15 min, and dehydration, transparency, sealing were observed under a microscope. 


\section{Average absorbance measurement}

Immunohistochemistry-positive cells or positive-stained region areas were measured, and then transformed from traditional subjective measurements to digitized measurements. Differences in the expression of aquaporins 1 and 3 between groups were analyzed using a semiquantitative method. Digital photos at 400X magnification were acquired using a high-definition microscopic image computer analysis system (Beijing Tech Instrument Co., Ltd., Version 5.0, Beijing, China) with the same microscope light intensity and under the manual setting condition. Each section was captured using 5 randomly selected visual fields. First, effective measurement region areas of pictures were measured; second, accumulated absorbance values of positive-stained cells in these regions were measured. Absorbance values were divided by the sample areas to calculate their averages. The peak static gray areas of pictures were measured, and all gray units of the program system were transformed to absorbance units. Effective measuring regions were selected to calculate average absorbance values for all recorded data.

\section{Main observation indicators}

The main observation indicators were conventional tissue pathology, immunohistochemistry, and average absorbance measurements.

For statistical analysis, normal distribution tests were conducted on all data using the Kolmogorov-Smirnov method with SPSS 16.0 (SPSS, Inc., Chicago, IL, USA). Normally distributed data with variance homogeneity were analyzed using a Student $t$-test, whereas normally distributed data without variance homogeneity were analyzed using an approximate $t$-test. Non-normally distributed data were analyzed using the rank sum test. All data are reported as means $\pm \mathrm{SD}$, and $\mathrm{P}<0.05$ indicated a significant difference.

\section{RESULTS}

\section{Conventional section observations under a light microscope}

\section{Control group}

Lumbar intervertebral disc tissue structures were clear with distinct collagen fiber shapes and slight edema but without mucoid degeneration (Figure 1).

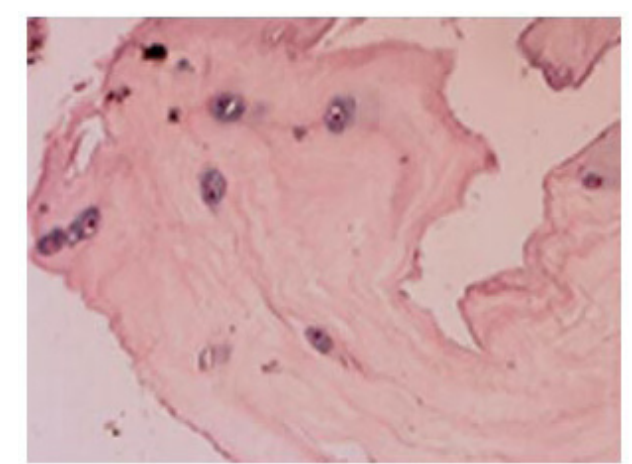

Figure 1. Lumbar intervertebral disc tissue in the control group (hematoxylin-eosin staining, 400X). 


\section{Disease group}

Tissue structures were obscure and disordered with hyperplastic collagen fibers and tissues of severe inflammatory edema and with necrosis mucoid degeneration. In the background of the fiber cartilage, the active cartilage cell number decreased or disappeared, and fibers were coarse and showed glassy degeneration. In nucleus pulposus tissues, cartilage-like cells displayed small nest shapes with disordered fiber hyperplasia and increased mucoid substances (Figure 2).

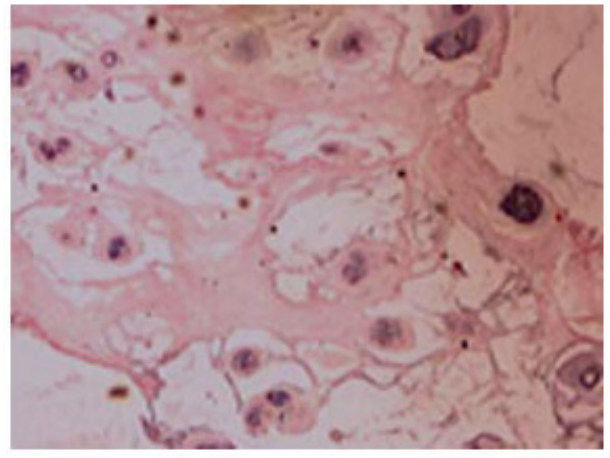

Figure 2. Lumbar intervertebral disc tissue in the lumbar intervertebral disc degeneration group (hematoxylin-eosin staining, 400X).

\section{Immunohistochemistry section observation}

Positive expression was indicated by brown or sepia cytoplasmic staining, whereas negative expression was indicated as the omission of a brown stain in the cytoplasm.

\section{Aquaporin 1 in the control group}

Immunohistochemistry images revealed positive expression in the annulus, cartilage endplate, and nucleus pulposus of the intervertebral disc. Almost no aquaporin 1 was expressed in the outer layer of the annulus (Figure 3).

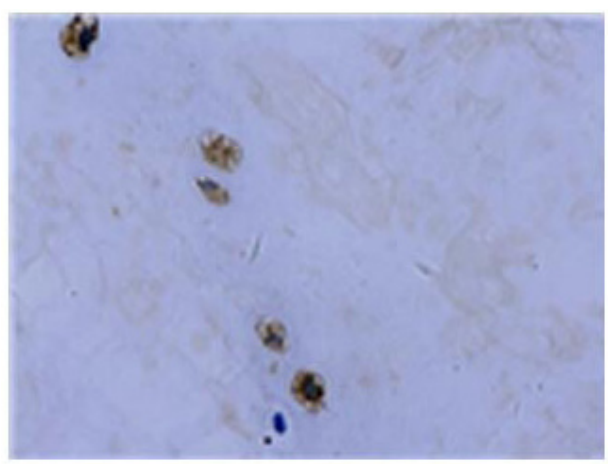

Figure 3. Immunohistochemistry of aquaporin 1 of the lumbar intervertebral disc tissue in the control group (streptavidin-peroxidase, 400X). 


\section{Aquaporin 1 in the disease group}

Immunohistochemistry images of the disease group revealed positive expression in intervertebral disc cells, but the color intensity was weaker than that in the control group, the positive cell number was lower, and expression was negative in most cells (Figure 4).

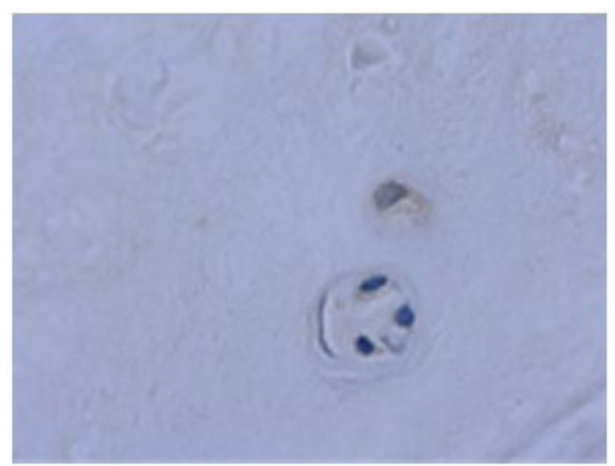

Figure 4. Immunohistochemistry of aquaporin 1 of the lumbar intervertebral disc tissue in the lumbar intervertebral disc degeneration group (streptavidin-peroxidase, 400X).

\section{Aquaporin 3 in the control group}

Immunohistochemistry images revealed positive expression of cells in the annulus, cartilage endplate, and nucleus pulposus of the intervertebral disc. Aquaporin 3 was expressed in all regions of the lumbar intervertebral disc, but it was expressed at higher levels in the nucleus pulposus (Figure 5).

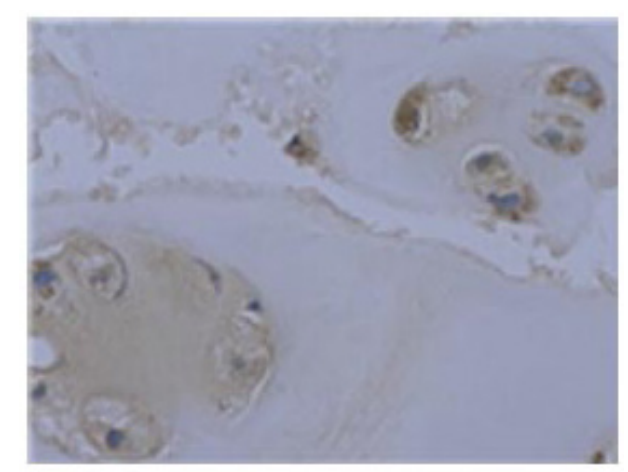

Figure 5. Immunohistochemistry of aquaporin 3 of the lumbar intervertebral disc tissue in the control group (streptavidin-peroxidase, 400X).

\section{Aquaporin 3 in the disease group}

Immunohistochemistry images of the disease group revealed positive expression cells in the intervertebral disc, but the color intensity was weaker than that in the control group, the positive cell number was lower, and expression was negative in most cells (Figure 6). 


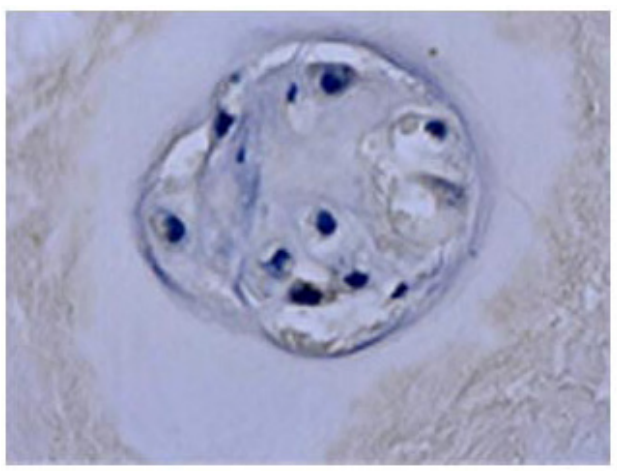

Figure 6. Immunohistochemistry of aquaporin 3 of lumbar intervertebral disc tissue in the lumbar intervertebral disc degeneration group (streptavidin-peroxidase, 400X).

\section{Average absorbance measurement}

There were significant differences between groups in average absorbance values of aquaporins 1 and 3 in the lumbar intervertebral disc (Table 1).

Table 1. Mean absorbance of aquaporins 1 and 3 in lumbar intervertebral disc tissue of control and degeneration groups.

\begin{tabular}{llr}
\hline Group & Aquaporin 1 & Aquaporin 3 \\
\hline Control & $0.701 \pm 0.424$ & $0.601 \pm 0.322$ \\
Intervertebral disc degeneration & $0.318 \pm 0.177^{\mathrm{a}}$ & $0.314 \pm 0.111^{\mathrm{a}}$ \\
\hline
\end{tabular}

Data are reported as means \pm SD for 15 individuals in each group. ${ }^{\mathrm{a}} \mathrm{P}<0.05 v s$ control group.

\section{DISCUSSION}

\section{Effect of various factors and periodization of lumbar intervertebral degeneration}

The mechanisms underlying lumbar intervertebral disc degeneration are unclear. Since many factors induce degeneration, it may be the result of the coordinated influence of various factors. Previous studies have suggested the following reasons for intervertebral degeneration: 1) the cartilage endplates of intervertebral discs degenerate and the fibroses and aquation degrees of the nucleus pulposus decrease, resulting in decreased intervertebral disc nutrition and viability; 2) stroma components degenerate and are modified to decrease the form and load-bearing capacities of intervertebral discs; 3 ) the mechanical load on the stroma of intervertebral discs are affected (Li and Guang, 2007).

As imaging technology has developed, periodization is conducted on intervertebral disc degeneration according to magnetic resonance imaging presentation. Thompson et al. (1990) divided intervertebral disc degeneration into 5 degrees: degree I, the nucleus pulposus resembles jelly, annuli are arranged in order, the thicknesses of transparent cartilage are well-distributed, and the centrum edges are regular; degree II, fiber tissues on the nucleus pulposus edges proliferate, mucus protein substances occur in the annulus, the thicknesses 
of transparent cartilages vary, and the centrum edges are irregular; degree III, fibroses occur in the nucleus pulposus, protein-like substances deposit in the annulus, spotty defects occur in transparent cartilage, and bony spurs grow on the centrum edges; degree IV, cracks appear in the nucleus pulposus, annuli display radials or splits, spotty defects in cartilage endplates calcify, and many bony spurs grow on centrum edges; degree V, cracks throughout entire intervertebral discs appear in the nucleus pulposus and annulus, cartilage endplates calcify widely, and the lengths of centrum bony spurs are longer than $2 \mathrm{~mm}$. Videman et al. (1994) divided degeneration into 4 degrees based on the variation in water content, but this periodization is rarely used in clinical applications. Pfirrmann et al. (2001) divided intervertebral degeneration into 5 degrees, and Griffith et al. (2007) improved this periodization. Because there is no publicly accepted standard regarding lumbar intervertebral disc periodization, additional studies classifying intervertebral disc degeneration, investigating degeneration mechanisms, and improving clinical therapy methods for intervertebral discs are necessary.

\section{Relationship of aquaporins and intervertebral disc degeneration}

Since Agre (2004) first identified aquaporins in red blood cells, more than 200 of these proteins have been identified, and 13 subtypes (aquaporins 0-12) were found in mammalian cells. Aquaporins are widely distributed in vivo and can be classified into 2 groups based on their specific functions: one that is characterized by highly selective water permeability (those that do not transport small molecular solutes other than water; aquaporins $0,1,2,4,5,6$, and 8), and another with relatively selective water permeability; aquaporins 3 and 7 are highly permeable to urea and glycerin, whereas aquaporin 9 is only permeable to urea (Hashido et al., 2005; Hedfalk et al., 2006; Hibuse et al., 2006; Magni et al., 2006).

Our results suggest that aquaporins 1 and 3 are closely correlated with the hydration of cartilage endplates, which is in concordance with their expression levels in the cartilage cells of the intervertebral disc. The high hydration degree and high permeability of the cartilage endplate expedite the transportation of nutrition and metabolism substances. The water content in the degenerative intervertebral disc is reduced and nutritional substances permeating into centrum cells are blocked, resulting in cell nutrition deficiency; this affects cell function and causes cell death. Cell death increases aquaporin 1 and 3 expression in the intervertebral disc to further block nutrition dispersion, resulting in a damaging cycle. Our results demonstrate that the expression of aquaporins 1 and 3 in intervertebral disc was significantly higher in control groups compared to disease groups. Therefore, reduced aquaporins 1 and 3 levels may be an important cause of lumbar intervertebral degeneration.

In conclusion, a common characteristic of factors that induce intervertebral disc degeneration is decreasing water content, and aquaporins 1 and 3 play important roles in lumbar intervertebral disc degeneration and may be initial factors in intervertebral disc degeneration. The inhibition of aquaporin 1 and 3 expression may offer a new method for curing and delaying intervertebral disc degeneration.

\section{REFERENCES}

Agre P (2004). Aquaporin water channels (Nobel Lecture). Angew. Chem. Int. Ed. Engl. 43: 4278-4290.

Cao GY, Yang YY, Jiang Y, et al. (2011). Intervertebral disc degenerative cartilage endplate AQP3 expression in the process of change. Chong Qing Yi Xue 40: 359-361. 
Chan D, Song Y, Sham P and Cheung KM (2006). Genetics of disc degeneration. Eur. Spine J. 15 (Suppl 3): S317-S325.

Dimcevici PN, Balalau C, Nimigean VR, Nimigean V, et al. (2014). Histopathological changes of renal tissue following sodium fluoride administration in two consecutive generations of mice. Correlation with the urinary elimination of fluoride. Rom. J. Morphol. Embryol. 55: 343-349.

Griffith JF, Wang YX, Antonio GE, Choi KC, et al. (2007). Modified Pfirrmann grading system for lumbar intervertebral disc degeneration. Spine 32: E708-E712.

Hashido M, Ikeguchi M and Kidera A (2005). Comparative simulations of aquaporin family: AQP1, AQPZ, AQP0 and GlpF. FEBS Lett. 579: 5549-5552.

Hedfalk K, Tornroth-Horsefield S, Nyblom M, Johanson U, et al. (2006). Aquaporin gating. Curr. Opin. Struct. Biol. 16: 447-456.

Hibuse T, Maeda N, Nagasawa A and Funahashi T (2006). Aquaporins and glycerol metabolism. Biochim. Biophys. Acta 1758: 1004-1011.

Li BJ and Guang XM (2007). The basis of the research progress on cervical disc degeneration. Zhong Guo Jiao Xing Wai Ke Za Zhi 15: 364-365.

Li SJ, Liang HJ, Ke CQ, et al. (2008). Lumbar disc protrusion and inflammatory factor change of nucleus pulposus with symptomatic pain. Zhong Guo Zu Zhi Gong Cheng Yan Jiu Yu Lin Chuang Kang Fu 12: 4303-4307.

Magni F, Sarto C, Ticozzi D, Soldi M, et al. (2006). Proteomic knowledge of human aquaporins. Proteomics 6: 5637-5649.

Nesic O, Lee J, Ye Z, Unabia GC, et al. (2006). Acute and chronic changes in aquaporin 4 expression after spinal cord injury. Neuroscience 143: 779-792.

Pfirrmann CW, Metzdorf A, Zanetti M, Hodler J, et al. (2001). Magnetic resonance classification of lumbar intervertebral disc degeneration. Spine 26: 1873-1878.

Richardson SM, Knowles R, Marples D, Hoyland JA, et al. (2008). Aquaporin expression in the human intervertebral disc. J. Mol. Histol. 39: 303-309.

Sobajima S, Kim JS, Gilbertson LG and Kang JD (2004). Gene therapy for degenerative disc disease. Gene Ther. 11: 390-401.

Takata K, Matsuzaki T and Tajika Y (2004). Aquaporins: water channel proteins of the cell membrane. Prog. Histochem. Cytochem. 39: 1-83.

Thompson JP, Pearce RH, Schechter MT, Adams ME, et al. (1990). Preliminary evaluation of a scheme for grading the gross morphology of the human intervertebral disc. Spine 15: 411-415.

Videman T, Nummi P, Battie MC and Gill K (1994). Digital assessment of MRI for lumbar disc desiccation. A comparison of digital versus subjective assessments and digital intensity profiles versus discogram and macroanatomic findings. Spine 19: 192-198.

Wang F and Zhu Y (2011). Effect of nucleus pulposus cells express water channel protein 1 experimental study of relevant factors. Zhong Hua Lin Chuang Yi Shi Za Zhi 5: 1634-1638.

Yang B, Ma T and Verkman AS (2001). Erythrocyte water permeability and renal function in double knockout mice lacking aquaporin-1 and aquaporin-3. J. Biol. Chem. 276: 624-628.

Yuan Y, Zhao J, Li YM, et al. (2011). Nuclear factor kappa B predominate, matrix metalloproteinases 3 expression in degenerative lumbar disc tissue. Zhong Guo Zu Zhi Gong Cheng Yan Jiu Yu Lin Chuang Kang Fu 15: 4111-4114. 